\title{
Increased Sodium Concentration in Substantia Nigra in Early Parkinson's Disease: A Preliminary Study With Ultra-High Field (7T) MRI
}

\section{OPEN ACCESS}

Edited by: Remy Guillevin,

Centre Hospitalier Universitaire ( $\mathrm{CHU})$ de Poitiers, France

Reviewed by:

Yasmin Blunck,

The University of Melbourne, Australia

Amgad Droby,

Tel Aviv Sourasky Medical

Center, Israel

*Correspondence:

Stephan Grimaldi

stephan.grimaldi@ap-hm.fr

Specialty section:

This article was submitted to Applied Neuroimaging,

a section of the journal

Frontiers in Neurology

Received: 27 May 2021

Accepted: 17 August 2021

Published: 09 September 2021

Citation:

Grimaldi S, El Mendili MM Zaaraoui W, Ranjeva J-P, Azulay J-P. Eusebio A and Guye M (2021) Increased Sodium Concentration in

Substantia Nigra in Early Parkinson's Disease: A Preliminary Study With

Ultra-High Field (7T) MRI

Front. Neurol. 12:715618.

doi: 10.3389/fneur.2021.715618

\author{
Stephan Grimaldi ${ }^{1,2,3 *}$, Mohamed Mounir El Mendili ${ }^{2,3}$, Wafaa Zaaraoui $i^{2,3}$, \\ Jean-Philippe Ranjeva ${ }^{2,3}$, Jean-Philippe Azulay ${ }^{1}$, Alexandre Eusebio ${ }^{1,4}$ and \\ Maxime Guye ${ }^{2,3}$
}

${ }^{1}$ APHM, Hôpital Universitaire Timone, Department of Neurology and Movement Disorders, Marseille, France, ${ }^{2}$ APHM, Hôpital Universitaire Timone, CEMEREM, Marseille, France, ${ }^{3}$ Aix Marseille Univ, CRMBM, CNRS, Marseille, France, ${ }^{4}$ Aix Marseille Univ, CNRS, Institut de Neurosciences de la Timone, Marseille, France

Pathophysiology of idiopathic Parkinson's disease (iPD) is complex and still misunderstood. At a time when treatments with disease-modifying potential are being developed, identification of early markers of neurodegeneration is essential. Intracerebral sodium accumulation could be one of them. Indeed, it may be in relation to the mitochondrial dysfunction that early exists in iPD. For the first time, we used brain sodium $\left({ }^{23} \mathrm{Na}\right) \mathrm{MRI}$ to explore sodium concentration changes that have already been reported to be related to neurodegeneration in other diseases. We prospectively included $10 \mathrm{iPD}$ patients (mean age $52.2 \pm 5.9$ years-old) with motor symptoms that started $<36$ months before inclusion and 12 healthy subjects (mean age $53 \pm 6.4$ years-old). Patients were scanned in OFF medication state by using proton $\left({ }^{1} \mathrm{H}\right)$ and ${ }^{23} \mathrm{Na} \mathrm{MRI}$ at $7 \mathrm{~T}$. We then extracted quantitative Total Sodium Concentration (TSC) from five regions of interest known to be early impaired in iPD [substantia nigra (SN), putamen, caudate nucleus, pallidum, thalamus] and in one region supposed to be relatively spared in the first stages of the disease [cortical gray matter (neocortex)]. Potential atrophy in these structures was also investigated with ${ }^{1} \mathrm{H}$ MRI. Relative to healthy subjects, iPD patients showed higher TSC in the SN (43.73 \pm 4.64 vs. $37.72 \pm 5.62, p=0.006$ after Bonferroni correction). A trend of increase in sodium concentrations was found within the pallidum $(45.80 \pm 4.19$ vs. $41.07 \pm 4.94, p=0.017$ ), putamen (48.65 \pm 4.58 vs. $43.66 \pm 5.04, p=0.041)$ and the cortical gray matter (56.34 \pm 3.92 vs. $50.81 \pm 5.50, p=0.021)$. No significant brain atrophy was found in patients compared to controls. Thus, alteration of sodium homeostasis in the $\mathrm{SN}$ in the absence of atrophy could be considered as a potential early marker of cellular dysfunction in iPD.

Keywords: Parkinson's disease, neurodegeneration, sodium, biomarker, ultra high field magnetic resonance imaging

\section{INTRODUCTION}

Idiopathic Parkinson's disease (iPD) is a neurodegenerative disease affecting the central nervous system and is a major cause of disability and dependence. By 2030, the number of parkinsonian patients could increase by 56\%, with 1 in 120 people over the age of 45 suffering from the disease (1). To date, there is no treatment to slow or stop the progression of the disease. At a time 
when treatments with disease-modifying potential are being developed (for example anti-synuclein antibodies or iron chelators) (2), identification of early markers of neurodegeneration is essential and these are urgently lacking.

Although $\alpha$-synuclein misfolding is part of a significant upstream pathway leading to dopaminergic degeneration $(3,4)$, mitochondrial dysfunction is another main upstream pathway to parkinsonism (5). Among the deleterious consequences of mitochondrial dysfunction, increased intracellular sodium $\left(\mathrm{Na}^{+}\right)$concentration has been reported (6) and reflects an alteration of the cellular homeostasis. Interestingly, $\mathrm{Na}^{+}$ concentration changes in the brain have already been related to neurodegeneration, for example in Huntington's Disease (7) or Amyotrophic Lateral Sclerosis (8) with brain sodium $\left({ }^{23} \mathrm{Na}\right) \mathrm{MRI}$ which allows the exploration of sodium distribution through a non-invasive procedure. Thus, we hypothesize that sodium accumulation could be a potential early biomarker of iPD, especially in the substantia nigra (SN) where impairment of regulation of mitochondrial DNA copy number occurs and is thought to be related to neurodegeneration in iPD (9). This pathophysiological approach could lead to the identification of new therapeutic targets such as the $\mathrm{Na}^{+} \mathrm{K}^{+} \mathrm{Cl}^{-}$cotransporter isoform 1 (NKCC1) importer antagonist bumetanide, which has been reported to attenuate motor effects of dopamine deprivation (10).

In this exploratory study, we propose to study brain sodium concentrations using ultra-high field (7T) MRI in iPD patients relative to healthy subjects.

\section{MATERIALS AND METHODS}

\section{Population Studied}

We prospectively recruited 10 patients with iPD between February and November 2020. All patients met the iPD MDS diagnostic criteria (11) with motor symptoms that started $<36$ months before inclusion.

Twelve age- and gender-matched healthy subjects were also recruited prospectively during the same period.

Written informed consent was obtained from all participants. The study was approved by the local Ethics Committee (Comité de Protection des Personnes Sud Méditerranée 1), in accordance with the Declaration of Helsinki.

\section{Clinical Data Collection}

All patients had been examined in a standardized manner within a half-day by a movement disorders specialist working in the Department of Neurology and Movement Disorders, Marseille University Hospital. We collected scores using different scales to evaluate the severity of symptoms [i.e., UPDRS III, Schwab \& England, Hoehn \& Yahr, SCOPA-AUT and REM-sleep behavior disorders (RBD) Screening Questionnaire (12)], cognition (MoCA, Lexical and semantic fluency, Benton Judgment of Line Orientation Test-15 items), mood disorder [Hospital Anxiety and Depression (HAD) scale] and apathy (Starkstein motivation scale). Levodopa equivalent dose was calculated (13). The clinical data are summarized in Table 1. After receiving the patient's agreement, dopaminergic treatment
TABLE 1 | Patients' characteristics.

\begin{tabular}{|c|c|c|}
\hline & Mean & SD \\
\hline Age (years) & 55.2 & 5.9 \\
\hline Disease duration (months) & 20.3 & 11.2 \\
\hline Schwab \& England & 97 & 4.8 \\
\hline Hoehn \& Yahr & 1 & 0 \\
\hline UPDRS III & 12.9 & 3.8 \\
\hline MoCA & 27.9 & 1.3 \\
\hline Total Lexical fluency (number in 2 min) & 20.2 & 7.4 \\
\hline Total Semantic fluecny (number in 2 min) & 28.8 & 3.9 \\
\hline Benton Test (15-items) & 30 & 2.7 \\
\hline Starkstein motivation scale & 19.9 & 5.7 \\
\hline $\mathrm{HAD}$ & 11.8 & 5.9 \\
\hline SCOPA-AUT & 9.7 & 7.8 \\
\hline RBD screen questionnaire & 3.7 & 2.2 \\
\hline Levodopa (md/day) & 80 & 188.9 \\
\hline \multirow[t]{2}{*}{ Levodopa Equivalent Dose (mg/day) } & 175.5 & 259.2 \\
\hline & \multicolumn{2}{|c|}{ Number of subjects (\%) } \\
\hline Gender & \multicolumn{2}{|c|}{$8 \mathrm{M}(80 \%)$ and $2 \mathrm{~F}(20 \%)$} \\
\hline Orthostatic hypotension & \multicolumn{2}{|c|}{$2(20 \%)$} \\
\hline Dopamine agonist & \multicolumn{2}{|c|}{$4(40 \%)$} \\
\hline Levodopa & \multicolumn{2}{|c|}{$3(30 \%)$} \\
\hline IMAO-B & \multicolumn{2}{|c|}{$6(60 \%)$} \\
\hline ICOMT & \multicolumn{2}{|c|}{$0(0 \%)$} \\
\hline Anticholinergic & \multicolumn{2}{|c|}{$0(0 \%)$} \\
\hline Levodopa Responsivness & \multicolumn{2}{|c|}{ Good for 10 (100\%) } \\
\hline
\end{tabular}

UPDRS, Unified Parkinson's Disease Rating Scale; MoCA, Montreal Cognitive Assessment; HAD, Hospital Anxiety and Depression scale; SCOPA-AUT, Scales for Outcomes in Parkinson's disease - AUTonomy; RBD, REM Sleep Behavior Disorder Screening Questionnaire; SD, standard deviation.

was suspended $72 \mathrm{~h}$ before scanning to evaluate the severity of the disease and levodopa responsiveness (good if $>50 \% / \mathrm{bad}$ ). Indeed, the effect of exogenous dopaminergic intake on a possible modification of sodium concentrations and metabolism is unknown.

\section{MRI Acquisition and Post-processing}

MRI acquisition was performed with a 7-T Magnetom system (Siemens, Erlangen, Germany). For ${ }^{23} \mathrm{Na}$ MRI exploration, we used a dual-tuned ${ }^{23} \mathrm{Na} /{ }^{1} \mathrm{H}$ QED birdcage coil and a multi-echo density adapted $3 \mathrm{D}$ projection reconstruction pulse sequence (TR $=120 \mathrm{~ms}, 5,000$ spokes, 384 radial samples per spoke, $3 \mathrm{~mm}$ nominal isotropic resolution; to ensure a sufficient number and distribution of TEs, while taking into account the $5 \mathrm{~ms}$ readout of the sequence, we applied the sequence three times within the same exam in order to obtain 24 TEs ranging from $0.2 \mathrm{~ms}$ to $70.78 \mathrm{~ms}$ : 1st acquisition: $0.20-9.70-19.20-28.70-38.20-$ 47.70 - 57.20 - $66.70 \mathrm{~ms}$, 2nd acquisition: 1.56 - $11.06-20.56$ - $30.06-39.56-49.06-58.56-68.06 \mathrm{~ms}$, 3rd acquisition: 4.28 - 13.78 - $23.28-32.78-42.28-51.78-61.28-70.78 \mathrm{~ms}$, total acquisition time $=3 \times 10 \mathrm{~min}$ ) adapted from (14). Six tubes with known sodium concentrations (from 25 to $100 \mathrm{mmol} / \mathrm{L}$ within $2 \%$ of agar gel) were placed within the field of view to 
TABLE 2 | Mean Total sodium concentration (TSC) (wet tissue volume, mmol/l) and normalized volumes of brain regions of interest.

\begin{tabular}{|c|c|c|c|c|c|}
\hline Brain areas & Group & $\mathrm{TSC} \pm \mathrm{SD}$ & $p$ & Normalized volume \pm SD & $p$ \\
\hline \multirow[t]{2}{*}{ Substantia Nigra } & iPD & $43.73 \pm 4.64$ & $0.006^{\star}$ & $0.07 \pm 0.01$ & 0.149 \\
\hline & Healthy subjects & $37.72 \pm 5.62$ & & $0.08 \pm 0.01$ & \\
\hline \multirow[t]{2}{*}{ Putamen } & iPD & $48.65 \pm 4.58$ & 0.041 & $0.62 \pm 0.14$ & 0.187 \\
\hline & Healthy subjects & $43.66 \pm 5.04$ & & $0.69 \pm 0.11$ & \\
\hline \multirow[t]{2}{*}{ Caudate nucleus } & iPD & $54.22 \pm 4.36$ & 0.055 & $0.48 \pm 0.07$ & 0.553 \\
\hline & Healthy subjects & $52.35 \pm 5.50$ & & $0.50 \pm 0.07$ & \\
\hline \multirow[t]{2}{*}{ Pallidum } & iPD & $45.80 \pm 4.19$ & 0.017 & $0.23 \pm 0.03$ & 0.461 \\
\hline & Healthy subjects & $41.07 \pm 4.94$ & & $0.24 \pm 0.04$ & \\
\hline \multirow[t]{2}{*}{ Thalamus } & iPD & $50.60 \pm 5.92$ & 0.129 & $0.97 \pm 0.14$ & 0.468 \\
\hline & Healthy subjects & $46.29 \pm 5.14$ & & $1.01 \pm 0.15$ & \\
\hline \multirow[t]{2}{*}{ Cortical Gray Matter } & iPD & $56.34 \pm 3.92$ & 0.021 & $42.53 \pm 5.67$ & 0.714 \\
\hline & Healthy subjects & $50.81 \pm 5.50$ & & $43.37 \pm 4.98$ & \\
\hline
\end{tabular}

*Survive after multiple comparisons. TSC, Total sodium concentration; SD, standard deviation; iPD, idiopathic Parkinson's Disease.

serve as a reference for quantification (14). A 32-channel phasedarray ${ }^{1} \mathrm{H}$ head coil $(1 \mathrm{Tx} / 32 \mathrm{Rx}$; Nova) was used to acquire a sub-millimeter ${ }^{1} \mathrm{H}$ three-dimensional Magnetization-Prepared 2 Rapid Acquisition Gradient-Echoes (MP2RAGE) sequence (TR $=5,000 \mathrm{~ms} / \mathrm{TE}=3 \mathrm{~ms} / \mathrm{TI} 1=900 \mathrm{~ms} / \mathrm{TI} 2=2,750 \mathrm{~ms}, 256$ slices, $0.6 \mathrm{~mm}$ isotropic resolution, acquisition time $=10 \mathrm{~min} 12 \mathrm{~s}$ ).

Sodium images were reconstructed offline, fitted using a biexponential model, and finally normalized relative to signal from reference tubes to obtain quantitative Total Sodium Concentration (TSC) maps of the whole brain. We followed the methodology previously described in (14). This multiecho approach allows to account for the complex relaxation processes of ${ }^{23} \mathrm{Na}$ through a bi-exponential fitting enabling to determine the ordinate at $\mathrm{TE}=0 \mathrm{~ms}$ of the curve corresponding to the TSC. In the present preliminary study with limited sample size and in order to minimize the statistical type II error, we decided to focus the analysis solely on TSC, the metric mainly expressed in the literature. Increase in sample size will allow to evaluate relative variations of short and long $\mathrm{T}^{*}$ pool fractions of ${ }^{23} \mathrm{Na}$. The first ${ }^{23} \mathrm{Na}$ echo $(\mathrm{TE}=$ $0.20 \mathrm{~ms}$ ) and the ${ }^{1} \mathrm{H}$ images were coregistered using a rigid transformation (15). ${ }^{1} \mathrm{H}$ images were segmented into Gray Matter (GM), White Matter (WM) and cerebrospinal fluid (CSF) (0.9 tissue probability threshold) using the Statistical Parametric Mapping 12 "New Segment" tool (16), into deep gray matter (DGM) using FSL-FIRST tool (accumbens, amygdala, caudate, hippocampus, pallidum, putamen and thalamus) (17) and into substantia nigra by registering the ${ }^{1} \mathrm{H}$ images to the Montreal Neurologic Institute (MNI) 152 space and bringing back the SN-AAL3 mask to the subject space (15). without resectioning (SPM8; https://www.fil.ion.ucl.ac.uk/spm/software/spm8/). The ${ }^{1} \mathrm{H}$ images were then segmented and normalized into the MNI template, and the resulting transformation was applied to the quantitative ${ }^{23} \mathrm{Na}$ maps. Quantitative TSC values were extracted from five regions of interest known to be early impaired in iPD (SN, putamen, caudate nucleus, pallidum, thalamus) and in one region supposed to be relatively spared in the first stages of the disease, the cortical gray matter (neocortex). Cerebral volumes were normalized for head size using the intracranial volume (18).

\section{Statistical Analysis}

Categorical variables are presented as numbers and percentages, and the quantitative results as a mean with standard deviation. Comparisons between groups were made with Chi2 or Fisher tests for categorical data and Wilcoxon for continuous data, as appropriate. Correlations between clinical features and MRI parameters were looked for with Spearman correlation analysis. A two-sided $p$-value $<0.05$ was considered statistically significant in univariate analysis and $p<0.008$ after Bonferroni correction. Statistical analyses were performed using JMP software JMP 9.0.1 (SAS Institute).

\section{RESULTS}

\section{Patients' and Healthy Subjects' Characteristics}

Eight males and 2 females with iPD were included in this study with a mean age of $55.2 \pm 5.9$ years-old and a mean disease duration of $20.3 \pm 11.2$ months. All of them were completely independent in their daily life as shown by scores of Schwab \& England $(97 \pm 4.8)$ and Hoehn \& Yahr $(1 \pm 0)$. No cognitive impairment was identified (MoCA score at $27.9 \pm 1.3$ ). Mean UPDRS III OFF anti-parkinsonian drugs was $12.9 \pm 3.8$. Clinical data are summarized in Table $\mathbf{1 .}$

iPD patients were comparable with healthy subjects concerning age (mean age of $53.0 \pm 6.4, p=0.35$ ) and gender $(6 \mathrm{~F}$ and $6 \mathrm{M}, p=0.20)$.

\section{Sodium Brain Accumulation and Brain Volume}

As shown in Table 2, Figures $\mathbf{1}$ and 2, relative to healthy subjects, patients with iPD showed higher TSC in the SN (43.73 \pm 4.64 vs. $37.72 \pm 5.62, p=0.006$ after Bonferroni correction). A trend of increase in sodium concentrations was found within the pallidum $(45.80 \pm 4.19$ vs. $41.07 \pm 4.94, p=0.017)$, putamen $(48.65 \pm 4.58$ 


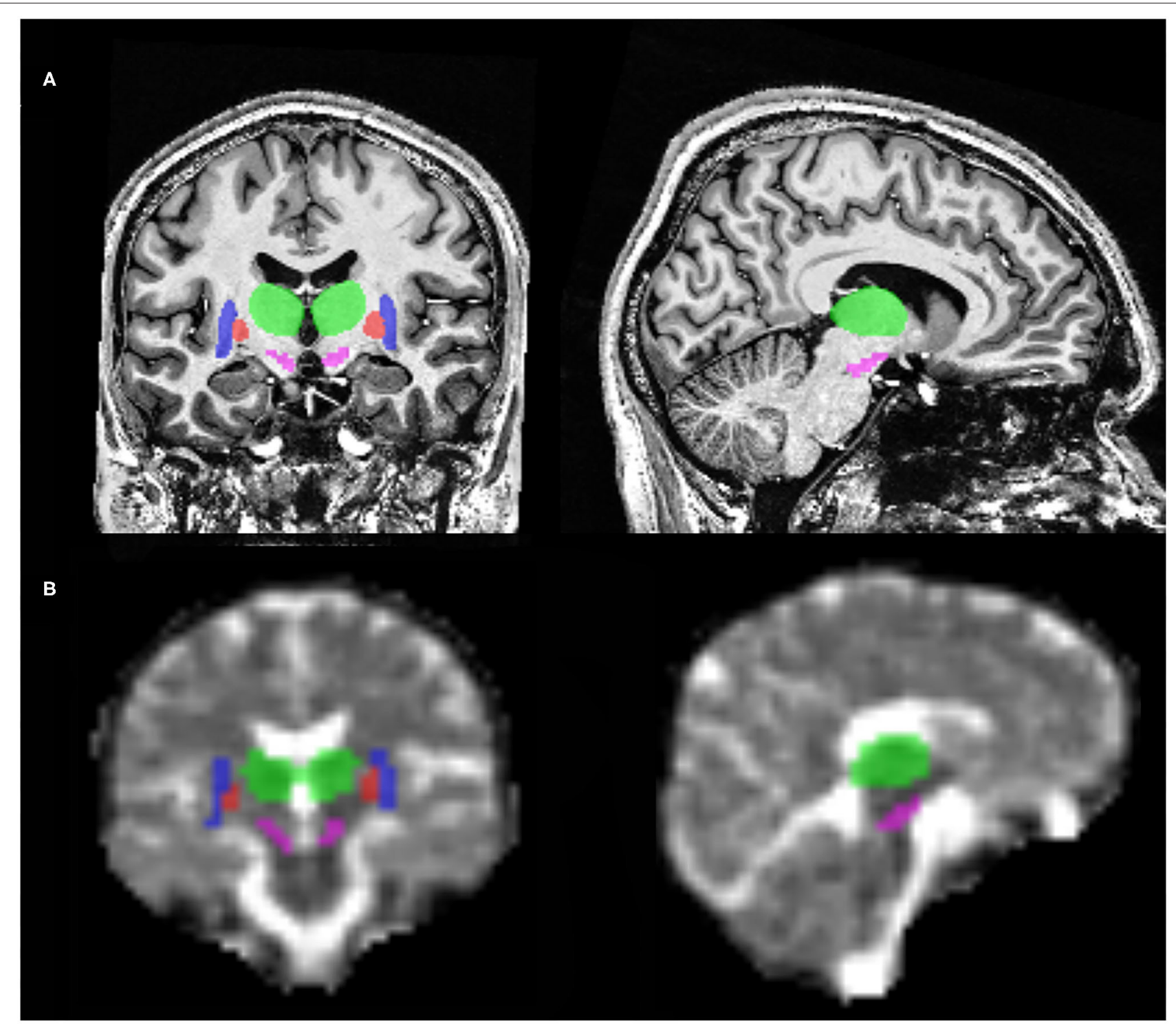

FIGURE 1 | Illustration of regions of interest with coronal and sagittal brain MRI slices at $7 \mathrm{~T}$. (A) ${ }^{1} \mathrm{H}$ T1-weighted MP2RAGE slices. (B) ${ }^{23}$ Na MRI slices. In this illustration, can be seen the substantia nigra (pink), putamen (blue), pallidum (red) and the thalamus (green).

vs. $43.66 \pm 5.04, p=0.041)$ and the cortical gray matter $(56.34 \pm$ 3.92 vs. $50.81 \pm 5.50, p=0.021)$.

No significant difference in ROI normalized volumes was found between patients and controls.

There was no correlation between clinical features, levodopa equivalent doses and MRI parameters.

\section{DISCUSSION}

Despite the limited number of subjects included in this study, we have been able to demonstrate an accumulation of sodium in patients with iPD. This accumulation is significant in the $\mathrm{SN}$ at this early stage of the disease.
$\mathrm{SN}$ is recognized as the origin of the nigro-basal gangliathalamic-cortical motor pathway dysfunction in iPD. Indeed, the most consistent finding of the neuropathology of iPD is a loss of dopaminergic neurons in the $\mathrm{SN}$ pars compacta which modulates activity of neurons in the basal ganglia. In the end, inadequate facilitation of the corticospinal tracts produces akinesia and bradykinesia which are part of parkinsonian syndrome (19). Pathophysiology of iPD is still misunderstood but along with $\alpha$ synuclein misfolding, mitochondrial dysfunction is considered to have an important role in neurodegeneration (5). Changes in mitochondrial gene expression, well-documented in the $\mathrm{SN}$ (9), seem to contribute to cellular energy failure, which in turn leads to loss of function of $\mathrm{Na}^{+} / \mathrm{K}^{+}$ATPase and impaired ability of the cell to maintain resting potential and to export $\mathrm{Na}^{+}(6)$. 


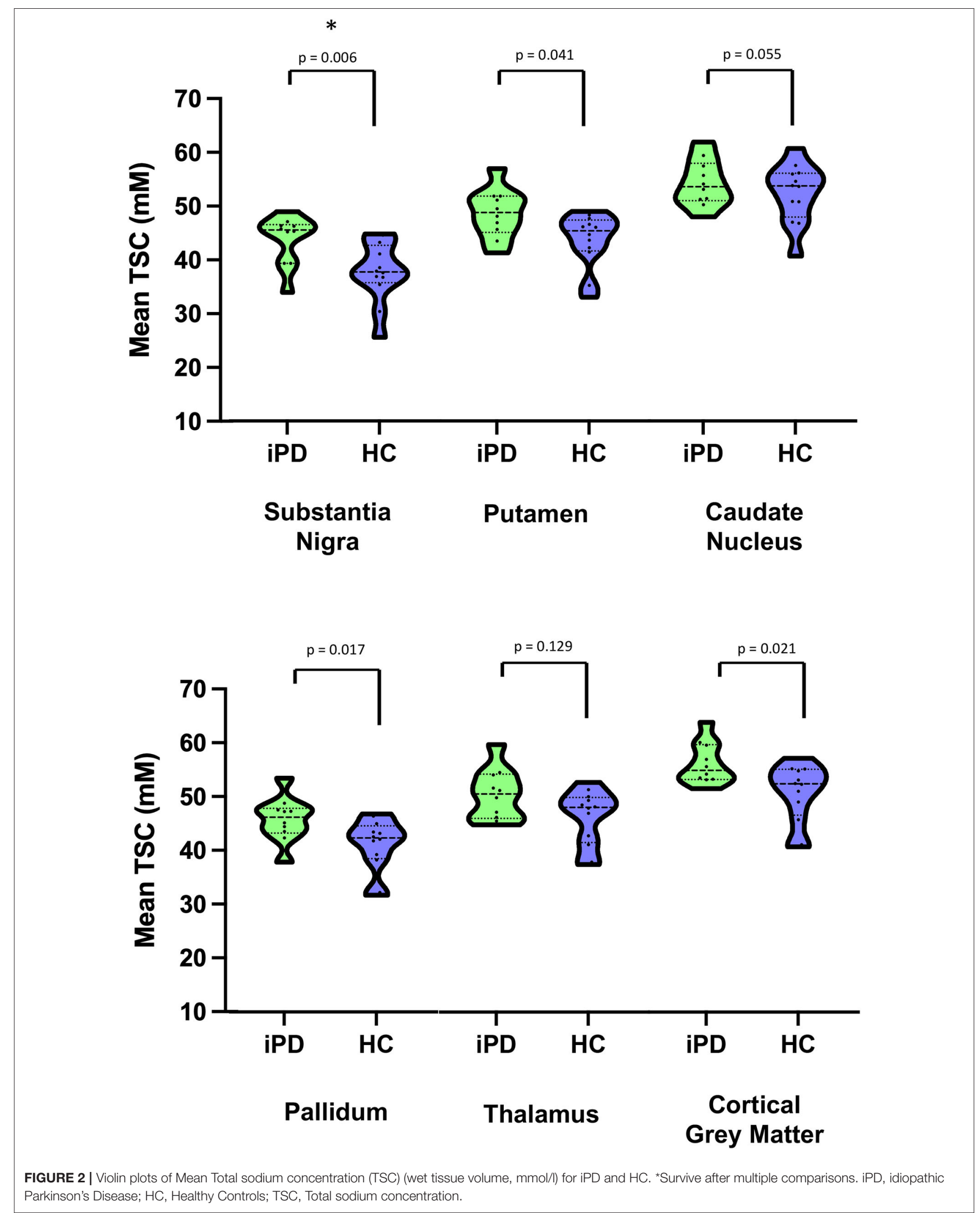


Then, the significant sodium accumulation in the $\mathrm{SN}$ is believed to be a sign of early dopaminergic neurons dysfunction in iPD. Also, the trend of increase in sodium concentrations within the pallidum corroborates the early SN-basal ganglia neural circuits impairment in iPD but more investigations are warranted to support these results.

The trend of sodium accumulation in cortical gray matter was not fully expected at this stage, in the absence of obvious cognitive impairment. However, early changes in personality and mild cognitive impairment have been described in iPD (20). Characteristically, these changes are often subtle at the beginning and difficult to detect without specific neuropsychological tests. Deficits mainly affect executive function including working memory and visuospatial capacity. Here, 3 patients had a MoCA score at $26 / 30$ which is the limit for normal values (21). Lewy pathology, hallmark of iPD, in the cerebral cortex does not correlate with cognitive impairment. Nevertheless, recent studies have shown abnormal mitochondria content and function, and increased oxidative stress and oxidative responses in the frontal cortex in iPD (22) that could be related to the tendency of sodium accumulation associated with early cortical dysfunction.

A variety of consequences of $\mathrm{Na}^{+}$accumulation may be relevant in PD pathophysiology. Mitochondrial dysfunction, ionic disturbances and neurodegeneration are interrelated biological processes already described iPD (5). Mitochondrial dysfunction may result in intraneuronal sodium overload through reversed activity of the sodium-calcium exchanger and axonal calcium import (6). In turn, this calcium overload may force the opening of mitochondrial permeability transition pore, leading to retrograde electron flux through the electron transport chain, resulting in increased reactive oxygen species production, release of cytochrome $c$, and activation of apoptosis $(23,24)$ particularly in SN dopaminergic neurons which project their axons on basal ganglia nuclei (25). We hypothesize that this accumulation of $\mathrm{Na}^{+}$may affect the activity of other structures in the SN-basal ganglia-thalamic-cortical circuit. For example, sodium ions are essential for spike generation in midbrain dopaminergic neurons (26) and pallidum neurons (27). A computational modeling study recently demonstrated a reduced availability of sub-threshold activated sodium and potassium channels, resulting in a decrease in firing regularity in pallidum neurons (28). Also, dopamine transporter (DAT) is a sodiumcoupled transmembrane protein that mediates the reuptake of dopamine from the synaptic cleft, and is localized to presynaptic nigrostriatal terminals (29). It operates by coupling transport of $\mathrm{Na}^{+}$along its concentration gradient from the extracellular compartment to the intracellular compartment allowing the transport of the substrate in the same way (30). Intracellular $\mathrm{Na}^{+}$overload might deteriorate its functioning and worsen neurological symptoms.

From a therapeutic point of view, interestingly, it has been shown that the neuronal $\mathrm{Na}^{+} \mathrm{K}^{+} \mathrm{Cl}^{-}$cotransporter isoform 1 (NKCC1) importer antagonist bumetanide, which reduces intracellular $\mathrm{Cl}^{-}$levels (but also reduces $\mathrm{Na}^{+}$and $\mathrm{K}^{+}$intracellular import) attenuates motor effects of dopamine deprivation by restoring GABAergic inhibition including the cortico-striatal pause-rebound response (31). Damier et al. (10), reported an improvement of iPD motor symptoms in the 4 patients treated with bumetanide which also improved gait and freezing in 2 of these patients calling for double-blind, placebocontrolled, randomized trials to confirm the therapeutic efficacy of bumetanide. NKCC1 importer antagonist has been postulated to have also a neuroprotective effect in $\mathrm{PD}$ through its action on astrocytes, microglia and oligodendrocytes by limiting the intracellular accumulation of $\mathrm{NA}^{+}, \mathrm{Cl}^{-}$and $\mathrm{K}^{+}$(32).

We did not find any significant atrophy when we compared the volumes of brain structures in iPD patients and healthy subjects although sodium concentration was abnormally elevated in patients. This suggests that abnormal elevated sodium concentration more likely reflects cellular dysfunction rather than cell death. This finding is in favor of the hypothesis that elevated sodium concentration could be a potential marker of early processes of neurodegeneration before neuronal death.

Our study has some limitations. The main one is the limited number of participants. This preliminary study has therefore allowed us to demonstrate the feasibility and relevance of assessing brain sodium accumulation in iPD. Unfortunately, several structures implicated in iPD pathophysiology are too small to be evaluated individually in sodium imaging such as locus coerulus, subthalamic-nucleus or SN pars compacta and SN pars reticulata separately due to the limited spatial resolution of sodium MRI even at high field.

\section{CONCLUSION}

To our knowledge, this is the first study using sodium MRI at 7T providing evidence of abnormal sodium concentration in $\mathrm{SN}$ which is a region known to be particularly impaired early in iPD. The evidence of such an alteration of sodium homeostasis in the absence of atrophy could be considered as a potential marker of early processes of cellular dysfunction and neurodegeneration before neuronal death in iPD. Further investigations are needed to confirm these results and to explore mapping of sodium homeostasis that might be different in other parkinsonian syndromes.

\section{DATA AVAILABILITY STATEMENT}

The raw data supporting the conclusions of this article will be made available by the authors, without undue reservation.

\section{ETHICS STATEMENT}

The studies involving human participants were reviewed and approved by Comité de Protection des Personnes Sud Méditerranée 1. The patients/participants provided their written informed consent to participate in this study.

\section{AUTHOR CONTRIBUTIONS}

SG: conception and design of the study, acquisition and analysis of data, drafting the manuscript and figures, and 
approval of final version of submitted manuscript. ME-M and J-PR: analysis of data, drafting the manuscript, approval of final version of submitted manuscript. WZ: analysis of data, drafting the manuscript, drafting the figure, approval of final version of submitted manuscript. J-PA, AE, and $\mathrm{MG}$ : conception and design of the study, drafting the manuscript, and approval of final version of submitted manuscript. All authors contributed to the article and approved the submitted version.

\section{REFERENCES}

1. Wanneveich M, Moisan F, Jacqmin-Gadda H, Elbaz A, Joly P. Projections of prevalence, lifetime risk, and life expectancy of Parkinson's disease (20102030) in France: projections of PD in France. Mov Dis. (2018) 33:144955. doi: 10.1002/mds.27447

2. Silva M, Caro V, Guzmán C, Perry G, Areche C, Cornejo A. $\alpha$-Synuclein and tau, two targets for dementia. Stud Natl Prod Chem. (2021) 67:125. doi: 10.1016/B978-0-12-819483-6.00001-1

3. Dauer W, Przedborski S. Parkinson's disease: mechanisms and models. Neuron. (2003) 39:889-909. doi: 10.1016/S0896-6273(03)00568-3

4. Schapira AH, Jenner P. Etiology and pathogenesis of Parkinson's disease. Mov Disord. (2011) 26:1049-55. doi: 10.1002/mds.23732

5. Abou-Sleiman PM, Muqit MMK, Wood NW. Expanding insights of mitochondrial dysfunction in Parkinson's disease. Nat Rev Neurosci. (2006) 7:207-19. doi: 10.1038/nrn1868

6. Waxman SG. Axonal conduction and injury in multiple sclerosis: the role of sodium channels. Nat Rev Neurosci. (2006) 7:932-41. doi: 10.1038/nr n2023

7. Reetz K, Romanzetti S, Dogan I, Saß C, Werner CJ, Schiefer $\mathrm{J}$, et al. Increased brain tissue sodium concentration in Huntington's disease - a sodium imaging study at 4T. NeuroImage. (2012) 63:517-24. doi: 10.1016/j.neuroimage.2012. 07.009

8. Grapperon A-M, Ridley B, Verschueren A, Maarouf A, ConfortGouny S, Fortanier E, et al. Quantitative brain sodium MRI depicts corticospinal impairment in amyotrophic lateral sclerosis. Radiology. (2019) 292:422-8. doi: 10.1148/radiol.20191 82276

9. Dölle C, Flønes I, Nido GS, Miletic H, Osuagwu N, Kristoffersen S, et al. Defective mitochondrial DNA homeostasis in the substantia nigra in Parkinson disease. Nat Commun. (2016) 7:13548. doi: 10.1038/ncomms 13548

10. Damier P, Hammond C, Ben-Ari Y. Bumetanide to treat Parkinson disease: a report of 4 cases. Clin Neuropharmacol. (2016) 39:57-59. doi: 10.1097/WNF.00000000000 00114

11. Postuma RB, Berg D, Stern M, Poewe W, Olanow CW, Oertel W, et al. MDS clinical diagnostic criteria for Parkinson's disease: MDS-PD clinical diagnostic criteria. Mov Disord. (2015) 30:1591-601. doi: 10.1002/mds. 26424

12. Stiasny-Kolster K, Mayer G, Schäfer S, Möller JC, Heinzel-Gutenbrunner M, Oertel WH. The REM sleep behavior disorder screening questionnaire-a new diagnostic instrument. Mov Disord. (2007) 22:2386-93. doi: 10.1002/mds. 21740

13. Schade S, Mollenhauer B, Trenkwalder C. Levodopa equivalent dose conversion factors: an updated proposal including opicapone and safinamide. Mov Disord Clin Pract. (2020) 7:343-5. doi: 10.1002/mdc3. 12921

14. Ridley B, Nagel AM, Bydder M, Maarouf A, Stellmann J-P, Gherib $\mathrm{S}$, et al. Distribution of brain sodium long and short relaxation times and concentrations: a multi-echo ultra-high field $23 \mathrm{Na}$ MRI study. Sci Rep. (2018) 8:4357. doi: 10.1038/s41598-018-22 711-0

\section{FUNDING}

This work was supported by ANR (ANR-15-CE19-0019-01, NEUROintraSOD-7T) and A*MIDEX (A*MIDEX-EI-17-29170228-09.43-Imetionic-7). Also, this work was performed by a laboratory member of France Life Imaging network (grant ANR-11-INBS-0006) and on the platform 7TAMI, a French Investissements dAvenir programme (grant ANR-11-EQPX-0001).

15. Avants BB, Tustison NJ, Stauffer $M$, Song G, Wu B, Gee JC. The insight ToolKit image registration framework. Front Neuroinform. (2014) 8:44. doi: 10.3389/fninf.2014. 00044

16. Ashburner J, Friston KJ. Unified segmentation. Neuroimage. (2005) 26:839-51. doi: 10.1016/j.neuroimage.2005. 02.018

17. Patenaude B, Smith SM, Kennedy DN, Jenkinson M. A bayesian model of shape and appearance for subcortical brain segmentation. Neuroimage. (2011) 56:907-22. doi: 10.1016/j.neuroimage.2011. 02.046

18. Barnes J, Ridgway GR, Bartlett J, Henley SMD, Lehmann M, Hobbs N, et al. Head size, age and gender adjustment in MRI studies: a necessary nuisance? Neuroimage. (2010) 53:1244-55. doi: 10.1016/j.neuroimage.2010. 06.025

19. Hutchison WD, Lozano AM, Davis KD, Saint-Cyr JA, Lang AE, Dostrovsky JO. Differential neuronal activity in segments of globus pallidus in Parkinson's disease patients. Neuroreport. (1994) 5:1533-7. doi: 10.1097/00001756-199407000-00031

20. Broeders M, de Bie RMA, Velseboer DC, Speelman JD, Muslimovic D, Schmand B. Evolution of mild cognitive impairment in Parkinson disease. Neurology. (2013) 81:346-52. doi: 10.1212/WNL.0b013e3182 $9 \mathrm{c} 5 \mathrm{c} 86$

21. Gill DJ, Freshman A, Blender JA, Ravina B. The montreal cognitive assessment as a screening tool for cognitive impairment in Parkinson's disease. Mov Disord. (2008) 23:1043-6. doi: 10.1002/mds.22017

22. Ferrer I. Early involvement of the cerebral cortex in Parkinson's disease: convergence of multiple metabolic defects. Prog Neurobiol. (2009) 88:89103. doi: 10.1016/j.pneurobio.2009.02.004

23. Mattson MP, Chan SL. Calcium orchestrates apoptosis. Nat Cell Biol. (2003) 5:1041-3. doi: 10.1038/ncb1203-1041

24. Ludtmann MHR, Abramov AY. Mitochondrial calcium imbalance in Parkinson's disease. Neurosci Lett. (2018) 663:8690. doi: 10.1016/j.neulet.2017.08.044

25. Surmeier DJ, Schumacker PT, Guzman JD, Ilijic E, Yang B, Zampese E. Calcium and Parkinson's disease. Biochem Biophys Res Commun. (2017) 483:1013-9. doi: 10.1016/j.bbrc.2016.08.168

26. Grace AA, Bunney BS. Intracellular and extracellular electrophysiology of nigral dopaminergic neurons-2. Action potential generating mechanisms and morphological correlates. Neuroscience. (1983) 10:317-31. doi: 10.1016/0306-4522(83)90136-7

27. Iyer R, Ungless MA, Faisal AA. Calcium-activated SK channels control firing regularity by modulating sodium channel availability in midbrain dopamine neurons. Sci Rep. (2017) 7:5248. doi: 10.1038/s41598-017-05578-5

28. Deister CA, Chan CS, Surmeier DJ, Wilson CJ. Calcium-activated SK channels influence voltage-gated ion channels to determine the precision of firing in globus pallidus neurons. J Neurosci. (2009) 29:8452-61. doi: 10.1523/JNEUROSCI.0576-09.2009

29. Borre L, Andreassen TF, Shi L, Weinstein H, Gether U. The second sodium site in the dopamine transporter controls cation permeation and is regulated by chloride. J Biol Chem. (2014) 289:25764-73. doi: 10.1074/jbc.M114.574269

30. Torres GE, Gainetdinov RR, Caron MG. Plasma membrane monoamine transporters: structure, regulation and function. Nat Rev Neurosci. (2003) 4:13-25. doi: 10.1038/nrn1008 
31. Lozovaya N, Eftekhari S, Cloarec R, Gouty-Colomer LA, Dufour A, Riffault B, et al. GABAergic inhibition in dual-transmission cholinergic and GABAergic striatal interneurons is abolished in Parkinson disease. Nat Commun. (2018) 9:1422. doi: 10.1038/s41467-018-0 $3802-\mathrm{y}$

32. Boscia F, Begum G, Pignataro G, Sirabella R, Cuomo O, Casamassa A, et al. Glial $\mathrm{Na}(+)$-dependent ion transporters in pathophysiological conditions. Glia. (2016) 64:1677-97. doi: 10.1002/glia. 23030

Conflict of Interest: The authors declare that the research was conducted in the absence of any commercial or financial relationships that could be construed as a potential conflict of interest.
Publisher's Note: All claims expressed in this article are solely those of the authors and do not necessarily represent those of their affiliated organizations, or those of the publisher, the editors and the reviewers. Any product that may be evaluated in this article, or claim that may be made by its manufacturer, is not guaranteed or endorsed by the publisher.

Copyright (๑) 2021 Grimaldi, El Mendili, Zaaraoui, Ranjeva, Azulay, Eusebio and Guye. This is an open-access article distributed under the terms of the Creative Commons Attribution License (CC BY). The use, distribution or reproduction in other forums is permitted, provided the original author(s) and the copyright owner(s) are credited and that the original publication in this journal is cited, in accordance with accepted academic practice. No use, distribution or reproduction is permitted which does not comply with these terms. 